\title{
In vitro antifungal activities of amphotericin B in
combination with acteoside, a phenylethanoid
glycoside from Colebrookea oppositifolia \\ Correspondence \\ Inshad Ali Khan \\ inshad2010@gmail.com \\ Received 4 March 2011 \\ Accepted 4 April 2011

\author{
Intzar Ali, ${ }^{1}$ Punita Sharma, ${ }^{2}$ Krishan Avtar Suri, ${ }^{2}$ Naresh Kumar Satti, ${ }^{2}$ \\ Prabhu Dutt, ${ }^{2}$ Farhat Afrin ${ }^{3}$ and Inshad Ali Khan ${ }^{1}$
} \\ ${ }^{1}$ Clinical Microbiology Division, Indian Institute of Integrative Medicine, Canal Road, Jammu Tawi 180001 , India
${ }^{2}$ Natural Product Chemistry Division, Indian Institute of Integrative Medicine, Canal Road, Jammu Tawi 180 001, India
${ }^{3}$ Department of Biotechnology, Faculty of Science, Hamdard University, Hamdard Nagar, New Delhi 110 062, India

\begin{abstract}
This study was undertaken to investigate the synergistic interaction between amphotericin $B$ $(\mathrm{AmB})$ and acteoside, isolated from the aerial parts of the shrub Colebrookea oppositifolia (Lamiaceae). Acteoside alone exhibited no intrinsic antifungal activity but showed a potent synergism in combination with AmB against selected pathogenic species, with fractional inhibitory concentration indices in the range of $0.0312-0.1562$. The combination of acteoside at 3.12 and $12.5 \mu \mathrm{g} \mathrm{ml}^{-1}$ with subinhibitory concentrations of AmB resulted in a potent fungicidal effect and also exhibited a significantly extended post-antifungal effect. Furthermore, the combination also reduced the minimum biofilm reduction concentration values of $A m B$ (2-16-fold) in preformed biofilms of Candida albicans, Cryptococcus neoformans and Aspergillus fumigatus. There was decreased viability of the cells, increased uptake of propidium iodide and enhanced leakage of $260 \mathrm{~nm}$-absorbing material by Candida albicans cells when exposed to AmB in the presence of acteoside. The reason for potentiation is likely to be that the subinhibitory concentrations of AmB facilitated the uptake of acteoside, which resulted in increased killing of the fungal cells.

Administration of acteoside in mice at up to $2000 \mathrm{mg}$ (kg body weight) ${ }^{-1}$ by the intraperitoneal or oral route produced no overt toxicity. The data presented here support synergism between acteoside and $\mathrm{AmB}$, and it is therefore proposed that a prospective new management strategy for
\end{abstract} therapeutic application of this combination should be explored.
}

\section{INTRODUCTION}

Invasive fungal infections have emerged as a major cause of morbidity and mortality in severely immunocompromised patients such as those undergoing chemotherapy or haematopoietic stem cell transplantation (Richardson, 2005). Candida species, Aspergillus species and Cryptococcus neoformans are among the most common aetiological agents of fungal infections (Pierce et al., 2008). Patients at risk of these invasive fungal infections are also at risk of developing other opportunistic infections, for which a wide range of non-antifungal therapeutic agents are used for prophylactic and therapeutic purposes concomitantly with antifungal agents (Marchetti et al., 2000; Afeltra \& Verweij, 2003;

Abbreviations: AmB, amphotericin B; FIC, fractional inhibitory concentration; MBRC, minimum biofilm reduction concentration; PAFE, postantifungal effect; PDA, potato dextrose agar; PI, propidium iodide; SDA, Sabouraud dextrose agar.
Antoniadou \& Giamarellou, 2007; Stergiopoulou et al., 2009). The economic cost of fungal infection and its associated mortality, especially in debilitated and highinvestment patients, remains unacceptably high (Wilson et al., 2002). Unfortunately, despite important new additions to the antifungal formulary such as third-generation broader-spectrum azoles and the more expensive echinocandins, as well as lipid formulations of amphotericin B $(\mathrm{AmB})$, in recent years, invasive fungal infections continue to be associated with mortality rates between $20 \%$ and nearly $100 \%$, depending on the infecting organism and the underlying condition of the patient (Nucci \& Perfect, 2008).

$\mathrm{AmB}$, a polyene macrolide antifungal antibiotic produced by Streptomyces nodosus, has been the 'gold standard' since its introduction in the 1950s for the treatment of invasive fungal infections. The classical and most frequently used formulation of this drug is AmB deoxycholate. However, it is associated with significant toxicity, including infusion-related events, 
such as chills, fever, headache, nausea and vomiting, and dose-limiting nephrotoxicity (Ostrosky-Zeichner et al., 2003). Newer lipid formulations of AmB with fewer adverse effects and activities similar to that of AmB deoxycholate have become available, but their high costs greatly limit their clinical use, especially in developing countries (Mariné et al., 2008). In the search for more effective chemotherapeutic approaches for treating invasive fungal infections, combination therapy is an important strategy, as synergistic interactions can potentially increase antifungal efficacy, reduce toxicity, provide a faster cure, prevent the emergence of resistance and provide broader-spectrum antifungal activity than monotherapy regimens (Marr et al., 2004).

In this regard, natural products are attractive prototypes in the search for new entities of plant origin that either have broad-spectrum antifungal activities or are modulators of the currently available antifungal agents; thus, these entities are focused mainly on a reduction in toxicity, enhancement of bioavailability, improvement of the antifungal spectrum and counteraction of resistance of existing drugs (Newman \& Cragg, 2007; Zhang et al., 2007; Hemaiswarya et al., 2008). There are several reports in the literature where natural products have demonstrated enhancement of antimicrobial activity (Khan et al., 2006; Stavri et al., 2007; Werle, 2008; Kumar et al., 2008; Lechner et al., 2008; Sharma et al., 2010). However, the number of natural compounds behaving synergistically with antifungals is minimal. This could be due to a limited understanding of the mechanism of action of drugs against these organisms or insufficient screening of natural compounds (Hemaiswarya et al., 2008).

Colebrookea oppositifolia (Lamiaceae) is a densely woolly shrub, distributed mostly in subtropical regions of the world such as India (CSIR, 1956) and China (Yang et al., 1996), and has been used extensively for the preparation of traditional herbal remedies for the treatment of fractures, traumatic injuries and rheumatoid arthritis (Chopra et al., 1956; Yang et al., 1996). The leaves of this plant are applied to wounds and bruises, and are used to relieve fever and headaches (Chopra et al., 1956). The juice of the young inflorescence is given to treat gastric problems and is also used in the nose for sinusitis (Malla \& Chhetri, 2009). A number of flavones and glycosides of flavones have been reported from the bark of this plant (Yang et al., 1996). Acteoside (verbascoside) (Fig. 1) is a phenylethanoid glycoside belonging to the group of watersoluble polyphenolic compounds, which are widely distributed in the plant kingdom and have been studied extensively for their various pharmacological activities such as hepatoprotective (Qazi et al., 2006), anti-inflammatory, antioxidant, antitumour, immunomodulatory, anti-nephritic, antiproliferative, antinociceptive, antimetastastic, antispasmodic, antibacterial, antiviral, neuroprotective, vasorelaxant and analgesic activities, improving sexual function, a sedative effect and strong radical scavenger activity (Díaz et al., 2004; Wu et al., 2007; Li et al., 2008).

Bearing in mind the excellent biological profile of acteoside and as a part of our ongoing anti-infective research (a)

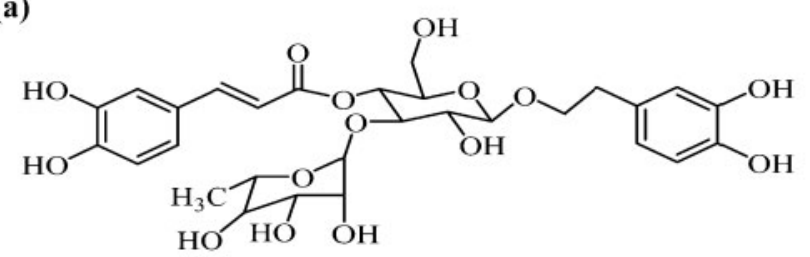

(b)

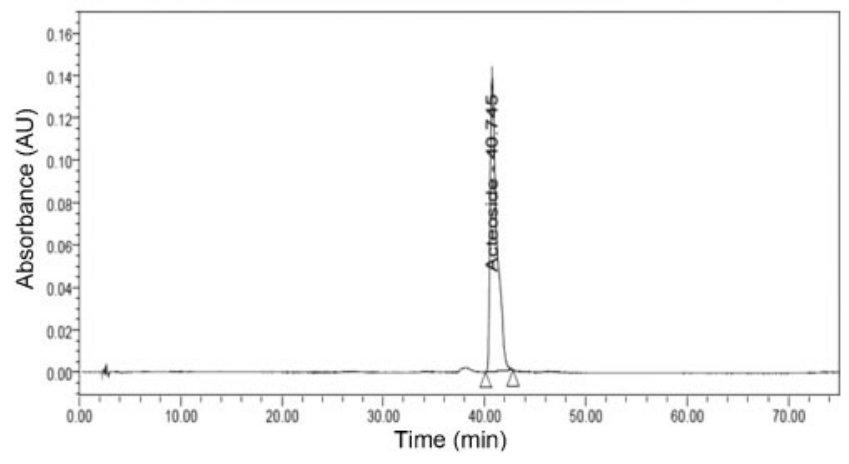

Fig. 1. Chemical structure (a) and HPLC chromatogram (b) of acteoside isolated from Colebrookea oppositifolia. AU, arbitrary units. The acteoside peak at 40.745 is labelled.

programme for potentiators of antimicrobial drugs, we studied the potentiating effect of acteoside with $\mathrm{AmB}$ in in vitro combination studies against opportunistic fungal pathogens. In addition, we evaluated its acute toxicological effect in mice.

\section{METHODS}

Extraction and isolation of acteoside from the aerial parts of Colebrookea oppositifolia. Shade-dried aerial parts of plant material of Colebrookea oppositifolia (500 g) were extracted four times in $50 \%$ aqueous ethanol $(4 \times 2.5 \mathrm{l})$ for $14 \mathrm{~h}$ each. The resulting extracts were pooled, filtered through muslin cloth, centrifuged and concentrated under reduced pressure at a temperature of $50 \pm 5{ }^{\circ} \mathrm{C}$ on a film evaporator. This concentrated extract was extracted three times with butanol in a separating funnel. The resulting butanol fractions were combined and concentrated under reduced pressure to yield a residue $(90 \mathrm{~g})$ containing $18 \%$ acteoside. The residue was subjected to adsorption chromatography using a silica gel column (200 g; 100200 mesh filter; $60 \times 3.2 \mathrm{~cm}$; Loba Chemie). The column was eluted with solvents by gradually increasing the percentage of methanol in chloroform. A total of 105 fractions of $100 \mathrm{ml}$ each were collected and pooled on the basis of thin-layer chromatography patterns checked using ethyl acetate/ $\mathrm{HCOOH} / \mathrm{H}_{2} \mathrm{O}(8: 1: 1$, by vol. $)$ as the mobile phase. Spots were visualized by spraying with freshly prepared borinate/polyethylene glycol 4000 solution [1\% solution of 2aminoethyl diphenylborinate in methanol and $5 \%$ solution of polyethylene glycol 4000 in ethanol, mixed $1: 1(\mathrm{v} / \mathrm{v})$ before spraying]. The $\mathrm{CHCl}_{3} /$ methanol $(5: 1, \mathrm{v} / \mathrm{v})$ elute was rechromatographed on a silica gel column using $\mathrm{CHCl}_{3} /$ methanol/ $\mathrm{H}_{2} \mathrm{O}(6: 1: 0.1$, by vol.) as solvent. The fractions that were homogeneous on thin-layer chromatography were pooled, dried and charged on a Sephadex LH-20 column and eluted with methanol to produce two fractions of 
$500 \mathrm{ml}$ each. The second fraction, containing acteoside, was subjected to further purification over a Sephadex LH-20 column. The column was eluted with methanol/ $\mathrm{H}_{2} \mathrm{O}(3: 2, \mathrm{v} / \mathrm{v})$ to produce a fraction that, on crystallization from methanol/chloroform, yielded a colourless amorphous powder soluble in methanol, with a flow rate of 0.42 [solvent system ethyl acetate/ $\mathrm{HCOOH} / \mathrm{H}_{2} \mathrm{O}(8: 1: 1$, by vol.)] and identified as acteoside (Fig. 1a) (Qazi et al., 2006).

The purity of the acteoside was established using a Waters HPLC system on a Merck RP- 18 column at $30^{\circ} \mathrm{C}$. The product was detected using a photodiode array detector at $335 \mathrm{~nm}$. The mobile phase consisting of acetonitrile/water and $1.5 \%$ acetic acid (17:83) was

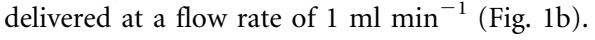

Fluorochrome dye. Propidium iodide (PI; Sigma), a small cationic, nucleic acid-binding fluorochrome largely excluded by intact cell membranes, was used to stain the yeast cells (Green et al., 1994). Sodium deoxycholate (Sigma), an anionic detergent, was used to facilitate diffusion of PI into the yeast cell membranes that were damaged by the antifungal agent (Joung et al., 2007).

Fungal strains and growth conditions. One reference strain and three clinical isolates of each of the following species were used for their in vitro susceptibility to $\mathrm{AmB}$ in combination with acteoside in this study: Candida albicans ATCC 90028, Candida glabrata ATCC 90030, Candida krusei ATCC 6258, Candida parapsilosis ATCC 22019, Candida tropicalis ATCC 750, Cryptococcus neoformans ATCC 204092, Aspergillus flavus MTCC 1973, Aspergillus fumigatus MTCC 1811, Aspergillus niger ATCC 16404 and Aspergillus parasiticus MTCC 2796. The reference strains were obtained from the American Type Culture Collection (ATCC) and Microbial Type Culture Collection (MTCC). The clinical isolates were obtained as a kind gift from Acharya Shri Chander (College of Medical Sciences, Sidhra, Jammu, India). The working strains were maintained on potato dextrose agar (PDA; Difco) slants at $4{ }^{\circ} \mathrm{C}$ and were subcultured twice prior to testing to ensure viability and purity.

Chequerboard antifungal susceptibility assay. The MIC of $\mathrm{AmB}$ was determined for yeast and Aspergillus species in the absence and presence of increasing concentrations of acteoside by a twodimensional chequerboard microbroth dilution method using twofold serial dilutions (Eliopoulus \& Moellering, 1996), following the Clinical and Laboratory Standards Institute guidelines (CLSI, 2008a, b). Antibiotic medium 3 (also known as Penassay broth; Difco) buffered to $\mathrm{pH} 7.0$ was used as a test medium. Stock solutions of AmB (Sigma) and acteoside were prepared in $100 \%$ DMSO (Sigma), with a final DMSO concentration of $1 \%(\mathrm{v} / \mathrm{v})$. Ten serial twofold dilutions of $\mathrm{AmB}$ were prepared in medium in amounts of $50 \mu \mathrm{l}$ per well in 96-well U-bottomed microtitre plates (Tarsons) to yield four times the final concentration required for testing, which ranged from 4 to $0.0078 \mu \mathrm{g} \mathrm{ml}^{-1}$. Seven serial twofold dilutions of acteoside were prepared separately in medium in microcentrifuge tubes to obtain four times the final concentration, ranging from 12.5 to $0.195 \mu \mathrm{g} \mathrm{ml}^{-1}$. Fifty microlitres of each acteoside concentration and its drug-free control was combined with $50 \mu \mathrm{l}$ of each concentration of $\mathrm{AmB}$ and its drug-free control in order to obtain a $12 \times 8$ chequerboard in 96-well U-bottomed microtitre plates. Stock inoculum suspensions of the yeasts and Aspergillus species were prepared in normal sterile saline containing $0.05 \%$ polysorbate 20 (HiMedia Laboratories) from $24 \mathrm{~h}(48 \mathrm{~h}$ for Cryptococcus neoformans) and 7 day cultures, respectively, grown on PDA at $35{ }^{\circ} \mathrm{C}$ (CLSI, 2008a, b). Inocula were verified for each assay by plating onto PDA plates for colony enumeration. These fungal suspensions were further diluted in Penassay broth and a $100 \mu \mathrm{l}$ volume of this diluted inoculum was added to each well of the plate, resulting in a final inoculum concentration of $0.5 \times 10^{4}-2.5 \times 10^{4}$ c.f.u. $\mathrm{ml}^{-1}$ for yeasts (Pfaller et al., 2004) and $0.4 \times 10^{4}-5 \times 10^{4}$ c.f.u. $\mathrm{ml}^{-1}$ for Aspergillus species (CLSI, 2008b). Growth-control, vehiclecontrol (DMSO) and sterility-control wells were included for each isolate tested. The plates were incubated at $35{ }^{\circ} \mathrm{C}$ for $48 \mathrm{~h}(72 \mathrm{~h}$ for Cryptococcus neoformans) and the wells were assessed visually for growth. The MIC was defined as the lowest concentration of the antifungal agent that completely prevented visible growth with respect to the growth control. The MIC of AmB was read alone and in the presence of different concentrations of acteoside. The synergistic interaction between $\mathrm{AmB}$ and acteoside for each strain was calculated based on the fractional inhibitory concentration (FIC) index, obtained by adding the FICs of AmB and acteoside. The FIC was calculated by dividing the MIC of the combination of $\mathrm{AmB}$ and acteoside by the MIC of AmB or acteoside alone (Berenbaum, 1978). An FIC index of $\leqslant 0.5$ indicated synergistic interactions, $\geqslant 0.5-4.0$ indicated no interaction and $\geqslant 4.0$ indicated antagonistic interactions (Odds, 2003). All experiments were conducted twice in duplicate on separate occasions with freshly prepared inocula and stock solutions.

Effect of acteoside on the fungicidal activities of AmB. The fungicidal effect of AmB in the presence and absence of acteoside on Candida albicans ATCC 90028, Cryptococcus neoformans ATCC 204092 and A. fumigatus MTCC 1811 was performed as described by Eliopoulus \& Moellering (1996) except that Penassay broth was used. The adjusted inoculum suspension $\left(\sim 5 \times 10^{6}\right.$ c.f.u. $\left.\mathrm{ml}^{-1}\right)$ was diluted tenfold in medium containing $\mathrm{AmB}$ at a concentration of $0.25 \times$ MIC $(0.125 \times$ MIC for Cryptococcus neoformans $)$ and in combination with increasing concentrations of acteoside ranging from 0.78 to $12.5 \mu \mathrm{g} \mathrm{ml}{ }^{-1}$. AmB alone at $4 \times$ MIC $(2 \times$ MIC for Cryptococcus neoformans) was used as a control. Culture flasks were incubated with an agitation of 200 r.p.m. at $35{ }^{\circ} \mathrm{C}$ for $24 \mathrm{~h}$ and the number of c.f.u. was determined on Sabouraud dextrose agar (SDA) plates with lecithin and polysorbate 80 (Becton Dickinson) using a serial dilution method. Plates were then incubated at $35^{\circ} \mathrm{C}$ for $24-$ $48 \mathrm{~h}$ (72 h for Cryptococcus neoformans). The lower limit of accurate and reproducible detectable colony counts was 10 c.f.u. $\mathrm{ml}^{-1}$. Synergy was defined as a 100-fold or greater decrease in colony count at $24 \mathrm{~h}$ by the combination of agents with reference to the starting inoculum and also when compared with the most active single agent (Hemaiswarya et al., 2008). All experiments were conducted in duplicate three times on separate occasions.

\section{Post-antifungal effect (PAFE) of the combination of AmB and} acteoside. The PAFE of AmB in the presence and absence of acteoside on Candida albicans ATCC 90028, and Cryptococcus neoformans ATCC 204092 was performed as described by Craig \& Gudmundsson (1996) except that Penassay broth was used. One millilitre of the adjusted inoculum suspension $\left(\sim 5 \times 10^{7}\right.$ c.f.u. $\left.\mathrm{ml}^{-1}\right)$ was added to $9 \mathrm{ml}$ Penassay broth medium with or without AmB alone and in combination with acteoside at a concentration of $3.12 \mu \mathrm{g} \mathrm{ml}^{-1}$, providing the starting inoculum of $\sim 5 \times 10^{6}$ c.f.u. $\mathrm{ml}^{-1}$. The AmB was tested at a concentration of $0.06 \mu \mathrm{g} \mathrm{m}^{-1}(0.25 \times \mathrm{MIC})$ for Candida albicans and $0.12 \mu \mathrm{g} \mathrm{ml}^{-1}(0.125 \times \mathrm{MIC})$ for Cryptococcus neoformans. After $2 \mathrm{~h}$ of exposure at $35{ }^{\circ} \mathrm{C}$ with agitation at 200 r.p.m., the samples were diluted 1:1000 in pre-warmed medium to effectively remove the AmB and acteoside. The diluted cultures were then incubated with agitation and sampling was carried out after $0,2,4,6,8,10,12,16,20,24,30$ and $48 \mathrm{~h}$ for c.f.u. enumeration. The c.f.u. counts were determined as described above for fungicidal activities. The PAFE was calculated using the following equation: $\mathrm{PAFE}=T-C$, where $T$ represents the time required for the count in the test culture to increase by $1 \log _{10}$ c.f.u. $\mathrm{ml}^{-1}$ above the count observed immediately after drug removal and $C$ represents the time required for the count of the untreated control flask to increase by $1 \log _{10}$ c.f.u. $\mathrm{ml}^{-1}$. Experiments were performed twice in duplicate. 
Inhibitory effect of a combination of AmB and acteoside on preformed fungal biofilms

Strains, growth conditions and inoculum. Candida albicans ATCC 90028, Cryptococcus neoformans ATCC 204092 and A. fumigatus MTCC 1811 were used for fungal biofilm formation. A stock inoculum suspension of yeasts was prepared from $24 \mathrm{~h}$ cultures grown in yeast peptone dextrose broth (Difco) (Pierce et al., 2008) and the cells were harvested in the late-exponential growth phase, followed by washing twice with sterile PBS (pH 7.4; Sigma), and the turbidity of the suspension was adjusted to a no. 5 McFarland standard $\left(\sim 5 \times 10^{7}\right.$ c.f.u. $\mathrm{ml}^{-1}$ for Candida albicans and $\sim 2 \times 10^{8}$ c.f.u. $\mathrm{ml}^{-1}$ for Cryptococcus neoformans). In the case of $A$. fumigatus, conidia were harvested for each experiment from fresh, mature (3-5 day) cultures grown on PDA slants by flooding the surface of the slants with $3 \mathrm{ml}$ normal sterile saline containing $0.05 \%$ polysorbate 20 and gently probing the surface with the tip of a Pasteur pipette. The resulting mixture of conidia and hyphal fragments was withdrawn and transferred to a sterile tube. This suspension was transferred to a sterile syringe attached to a sterile filter with a pore diameter of $11 \mu \mathrm{m}$ (Millipore), filtered and collected in a sterile tube. The density of the suspension was adjusted to $1 \times 10^{6}-5 \times 10^{6}$ c.f.u. $\mathrm{ml}^{-1}$. Each suspension was quantified by plating on PDA plates and the standardized suspension was used immediately.

Minimum biofilm reduction concentration (MBRC). The effect of a combination of $\mathrm{AmB}$ and acteoside on preformed biofilms was examined by a two-dimensional chequerboard broth microdilution method (Eliopoulus \& Moellering, 1996), as described previously (Pierce et al., 2008). The adjusted fungal suspensions were diluted further to a final density of $\sim 1 \times 10^{6}$ c.f.u. $\mathrm{ml}^{-1}$ for Candida albicans and $\sim 1 \times 10^{7}$ c.f.u. $\mathrm{ml}^{-1}$ for A. fumigatus in RPMI 1640 containing Lglutamine without sodium bicarbonate and buffered to $\mathrm{pH} 7.0$ with $0.165 \mathrm{M}$ morpholinepropanesulfonic acid (both from Sigma) (CLSI, 2008a, b), whilst for Cryptococcus neoformans, $\sim 1 \times 10^{7}$ c.f.u. $\mathrm{ml}^{-1}$ was used in Dulbecco's modified Eagle's medium (Sigma) (Pierce et al., 2008). A $200 \mu \mathrm{l}(100 \mu \mathrm{l}$ for Candida albicans) volume of these suspensions was inoculated into selected wells of a 96-well flatbottomed polystyrene microtitre plate (Nunc). After incubation at $35{ }^{\circ} \mathrm{C}$ for $24 \mathrm{~h}$ ( $48 \mathrm{~h}$ for Cryptococcus neoformans), the culture supernatant from each well was decanted and the planktonic cells were removed by washing the wells with PBS. Twofold serial dilutions of AmB (32-0.03 $\mu \mathrm{g} \mathrm{ml}^{-1}$ ) and acteoside (25-6.25 $\mu \mathrm{g} \mathrm{ml}^{-1}$ ) were prepared in Penassay broth to yield twice the final concentration required for testing and a $100 \mu \mathrm{l}$ aliquot of each dilution from both the agents were added to the selected wells containing the biofilm. The plate was further incubated at $35{ }^{\circ} \mathrm{C}$ for $24 \mathrm{~h}$. In this assay, medium without the agents was used as the non-treated control and medium alone as the blank control (four wells of each microtitre plate). After incubation, the plates were decanted and washed three times with $200 \mu \mathrm{l}$ sterile PBS to remove loosely adherent cells. The biofilm reduction was quantified using an XTT reduction assay as described below. The experiment was performed three times with two replicates on separate occasions.

Measurement of biofilm metabolic activity by XTT reduction assay. A semi-quantitative measurement of biofilm was obtained using an XTT [tetrazolium salt; sodium 2,3-bis-(2-methoxy-4-nitro-5sulfophenyl)-2H-tetrazolium-5-carboxanilide; Sigma) reduction assay, performed by the method of Pierce et al. (2008). Briefly, XTT solution was prepared in PBS $\left(0.5 \mathrm{mg} \mathrm{ml}^{-1}\right)$, filter sterilized through a $0.22 \mu \mathrm{m}$ filter (Millipore) and stored at $-80{ }^{\circ} \mathrm{C}$ until required. Prior to each assay, menadione solution (10 $\mathrm{mM}$ prepared in acetone; Sigma) was filtered and mixed with XTT solution (thawed) at a final concentration of $1 \mu \mathrm{M}$ for Candida albicans and $10 \mu \mathrm{M}$ for Cryptococcus neoformans and A. fumigatus. XTT/menadione solution $(100 \mu \mathrm{l})$ was added to each of the pre-washed wells and the plates were incubated in the dark for $2-3 \mathrm{~h}$ at $35^{\circ} \mathrm{C}$. Following incubation, $75 \mu \mathrm{l}$ of the solution was transferred to a fresh microtitre plate and the colour change in the solution was measured spectrophometrically at $490 \mathrm{~nm}$ using a microtitre plate reader (Multiskan Spectrum; Thermo Scientific).

Cell viability assay of AmB and acteoside combination. The viability of Candida albicans ATCC 90028 following treatment with $\mathrm{AmB}$ in the presence and absence of acteoside was determined by a serial dilution method as described above for the fungicidal activities of AmB, with some modification. A cell suspension of Candida albicans in sterile MilliQ water $\left(1 \mathrm{ml}, \sim 5 \times 10^{7}\right.$ c.f.u. $\left.\mathrm{ml}^{-1}\right)$ was exposed to a subinhibitory concentration of $\mathrm{AmB}\left(0.25 \mu \mathrm{g} \mathrm{ml} \mathrm{m}^{-1}\right)$ alone and in combination with a fixed concentration of acteoside $\left(25 \mu \mathrm{g} \mathrm{ml}^{-1}\right)$ for $120 \mathrm{~min}$ under agitation in a dark chamber at $35^{\circ} \mathrm{C}$. AmB was also tested alone at a concentration of $4.0 \mu \mathrm{g} \mathrm{ml}^{-1}$. After exposure, the samples were serially diluted in sterile normal saline containing $0.1 \%$ polysorbate 80 (Sigma) for the inactivation of AmB. A $100 \mu \mathrm{l}$ aliquot was plated onto SDA plates containing lecithin and polysorbate 80 . Colonies were counted after $48 \mathrm{~h}$ incubation at $35{ }^{\circ} \mathrm{C}$ and the viable cell number was reported as c.f.u. $\mathrm{ml}^{-1}$.

PI uptake assay for AmB in combination with acteoside. The disruptive effect of AmB in the presence and absence of acteoside on Candida albicans ATCC 90028 cell membranes was assessed by AmBmediated PI uptake. Cell suspensions of Candida albicans in sterile MilliQ water $\left(1 \mathrm{ml}, \sim 5 \times 10^{7}\right.$ c.f.u. $\left.\mathrm{ml}^{-1}\right)$ were incubated with a subinhibitory concentration of $\mathrm{AmB}\left(0.25 \mu \mathrm{g} \mathrm{ml}^{-1}\right)$ alone and in combination with a fixed concentration of acteoside $\left(25 \mu \mathrm{g} \mathrm{ml}^{-1}\right)$ for 120 min under agitation in a dark chamber at $35^{\circ} \mathrm{C}$. Ten minutes prior to the completion of incubation, $10 \mu \mathrm{l}$ each of PI and sodium deoxycholate solution was added at final concentrations of $25 \mu \mathrm{g} \mathrm{ml}^{-1}$ and $2.5 \mathrm{mg} \mathrm{ml}^{-1}$, respectively (Green et al., 1994; Joung et al., 2007). $\mathrm{AmB}$ was also tested alone at a concentration of $4.0 \mu \mathrm{g} \mathrm{ml}^{-1}$. Cells without $\mathrm{AmB}$ and acteoside served as the negative (growth) control and were treated in a similar fashion. After incubation, $50 \mu \mathrm{l}$ was transferred to a fluorescence-activated cell sorting tube (Becton Dickinson) containing $950 \mu \mathrm{l}$ sterile MilliQ water. Each tube was analysed using a FACSCalibur (Becton Dickinson) with CellQuest Pro software for data acquisition and analysis. Each experiment was conducted three times in duplicate on separate occasions.

Leakage of $\mathbf{2 6 0} \mathbf{n m - a b s o r b i n g ~ m a t e r i a l ~ o n ~ e x p o s u r e ~ t o ~ A m B ~ i n ~}$ combination with acteoside. Exponentially growing cells of Candida albicans ATCC $90028\left(\sim 5 \times 10^{7}\right.$ c.f.u. $\left.\mathrm{ml}^{-1}\right)$ were suspended in sterile MilliQ water and exposed to a subinhibitory concentration of $\mathrm{AmB}\left(0.25 \mu \mathrm{g} \mathrm{ml}^{-1}\right)$ alone and in combination with a fixed concentration of acteoside $\left(25 \mu \mathrm{g} \mathrm{ml}^{-1}\right)$ for $120 \mathrm{~min}$ at $35{ }^{\circ} \mathrm{C}$ with agitation at 100 r.p.m. in a dark chamber. After centrifugation (17 $968 \mathrm{~g}$ at $4{ }^{\circ} \mathrm{C}$ for $10 \mathrm{~min}$ ), the absorbance of the supernatants was read in a UV spectrophotometer (Specord UV-250; Analytic Jena AG) at $260 \mathrm{~nm}$. Cells exposed to $\mathrm{AmB}$ at a concentration of $4 \mu \mathrm{g} \mathrm{ml}^{-1}$ followed by sonication with a probe sonicator (six cycles of $1 \mathrm{~min}$ each) served as the positive control. The unexposed cell suspension was used as a negative control. The experiment was conducted twice in duplicate on separate occasions.

Acute toxicity studies of acteoside. The maximum tolerable dose of acteoside was determined intraperitoneally and orally. The acute toxicity studies were carried out following the Organization for Economic Co-operation and Development guidelines (OECD, 2006) after approval from the Institutional Animal Ethics Committee (IAEC study no. SSP-0408, March 2009). Swiss albino mice 6-8 weeks old with a body weight of $22-30 \mathrm{~g}$ in groups of ten mice of both sexes were used, obtained from the institute's animal house. All animals were housed in standard-sized polycarbonate cages with controlled conditions of temperature $\left(23 \pm 1{ }^{\circ} \mathrm{C}\right)$ and humidity $(55 \pm 10 \%)$ and 
a $12: 12 \mathrm{~h}$ light: dark cycle; they were fed a standard pellet diet (Gold Muhor; Lipton India) and filtered water was provided ad libitum. A stock solution of acteoside was prepared in $10 \%$ ethanol (Merck) prior to the experiment and administered to overnight-fasted mice intraperitoneally or orally with a single-bolus dose in the range of 100-2000 mg (kg body weight $)^{-1}$. The mice were administered different doses of the test compound and observed individually after dosing at least once during the first $30 \mathrm{~min}$ and periodically for the first $24 \mathrm{~h}$. Experiments were performed three times on separate occasions

Statistical analysis. All experiments were repeated two or three times with two replicates for each condition tested and similar results were obtained on all occasions. The results are expressed as the mean $\pm \mathrm{SD}$, and statistical analysis was carried out using Student's $t$ test and one-way analysis of variance (Dunnett's multiple comparison test), using Excel 2003 (Microsoft). $P<0.05$ was considered to be statistically significant.

\section{RESULTS}

\section{Evaluation of acteoside for potentiation of AmB activity using a chequerboard assay}

The MIC of $\mathrm{AmB}$ was measured in the absence and presence of increasing concentrations of acteoside (0.195$12.5 \mu \mathrm{g} \mathrm{ml}^{-1}$ ) against six reference strains of yeasts and four reference strains of Aspergillus species (all clinically significant fungal pathogens) using a two-dimensional chequerboard microbroth dilution method. Acteoside on its own did not show any antifungal activity when tested at concentrations up to $1000 \mu \mathrm{g} \mathrm{ml}^{-1}$. However, interestingly, it showed a potent synergism in combination with $\mathrm{AmB}$ against all tested fungal species, with FIC indices in the range of $0.0312-0.1562$ (Table 1). The FIC of AmB when combined with acteoside ranged from 0.0156 to 0.125 for yeasts (8-64-fold reduction in AmB MIC) and was 0.125 for Aspergillus species (eightfold reduction in AmB MIC). A more prominent synergistic interaction between $\mathrm{AmB}$ and acteoside was observed for Cryptococcus neoformans (FIC index 0.0312: 64-fold reduction in AmB MIC), among all the fungal species tested. DMSO (1\%, vehicle control) had no inhibitory effect on the growth of the tested fungal species when compared with the growth control.

\section{Effect of acteoside on the fungicidal activities of AmB}

The fungicidal effect of the combination of $A m B$ with acteoside was assessed on Candida albicans ATCC 90028, A. fumigatus MTCC 1811 and Cryptococcus neoformans ATCC 204092. AmB was used at a concentration of $0.25 \times$ MIC $(0.125 \times$ MIC for Cryptococcus neoformans $)$, as well as in combination with increasing concentrations of acteoside ranging from 0.78 to $12.5 \mu \mathrm{g} \mathrm{ml}^{-1}$. As expected, $\mathrm{AmB}$ alone at these concentrations did not show any inhibitory activity, whilst the fungicidal activity (99.9\% kill) was achieved at $4 \times$ MIC $(2 \times$ MIC for Cryptococcus neoformans) in $24 \mathrm{~h}$ when compared with the growth

Table 1. In vitro synergic effect of acteoside (AS) on the activity of amphotericin B (AmB) against ten reference strains of selected fungi as determined by the chequerboard broth microdilution method following CLSI guidelines by using antibiotic medium 3 (Penassay broth)

The MIC (minimum inhibitory concentration) of Candida species was determined by using higher range of inoculum (Pfaller et al., 2004). Three clinical isolates of each species were also tested and no statistically significant changes were observed (data not shown). Results are representative of two separate experiments performed in duplicate with similar results each time.

\begin{tabular}{|c|c|c|c|c|c|c|c|}
\hline \multirow[t]{2}{*}{ Tested species } & \multicolumn{3}{|c|}{ AmB MIC $\left(\mu \mathrm{g} \mathrm{ml}^{-1}\right)$} & \multicolumn{3}{|c|}{ AS MIC ( $\left.\mu \mathrm{g} \mathrm{ml}^{-1}\right)$} & \multirow{2}{*}{$\begin{array}{c}\text { FIC index } \\
\left(\text { FIC }_{\mathrm{A}}+\text { FIC }_{\mathrm{B}}\right)\end{array}$} \\
\hline & AmB alone & $\begin{array}{l}\text { AmB combined } \\
\text { with AS }\end{array}$ & AmB FIC $\left(\right.$ FIC $\left._{\mathrm{A}}\right)$ & AS alone & $\begin{array}{l}\text { AS combined } \\
\text { with AmB }\end{array}$ & AS FIC $\left(\right.$ FIC $\left._{B}\right)$ & \\
\hline $\begin{array}{l}\text { Candida } \\
\text { albicans }\end{array}$ & 0.25 & 0.0156 & 0.0624 & $>12.5$ & $<0.195$ & 0.0156 & 0.078 \\
\hline $\begin{array}{l}\text { Candida } \\
\text { glabrata }\end{array}$ & 0.5 & 0.0625 & 0.125 & $>12.5$ & 0.39 & 0.0312 & 0.1562 \\
\hline Candida krusei & 0.25 & 0.0156 & 0.0624 & $>12.5$ & $<0.195$ & 0.0156 & 0.078 \\
\hline $\begin{array}{l}\text { Candida } \\
\text { parapsilosis }\end{array}$ & 0.5 & 0.0625 & 0.125 & $>12.5$ & 0.39 & 0.0312 & 0.1562 \\
\hline $\begin{array}{l}\text { Candida } \\
\text { tropicalis }\end{array}$ & 0.5 & 0.0312 & 0.0624 & $>12.5$ & $<0.195$ & 0.0156 & 0.078 \\
\hline $\begin{array}{c}\text { Cryptococcus } \\
\text { neoformans }\end{array}$ & 1.0 & 0.0156 & 0.0156 & $>12.5$ & $<0.195$ & 0.0156 & 0.0312 \\
\hline A. flavus & 2.0 & 0.25 & 0.125 & $>12.5$ & 0.39 & 0.0312 & 0.1562 \\
\hline A. fumigatus & 0.5 & 0.0625 & 0.125 & $>12.5$ & 0.39 & 0.0312 & 0.1562 \\
\hline A. niger & 1.0 & 0.125 & 0.125 & $>12.5$ & 0.39 & 0.0312 & 0.1562 \\
\hline A. parasiticus & 1.0 & 0.125 & 0.125 & $>12.5$ & 0.39 & 0.0312 & 0.1562 \\
\hline
\end{tabular}


control $(P<0.001)$. However, the same subinhibitory concentrations of $\mathrm{AmB}$ resulted in fungicidal activity when tested in combination with acteoside at concentrations of $0.78-12.5 \mu \mathrm{g} \mathrm{ml}^{-1}$ for $24 \mathrm{~h}$ (Fig. 2). The fungicidal activity of the combination was equivalent to the fungicidal activity of $\mathrm{AmB}$ alone at $4 \times \mathrm{MIC}(2 \times \mathrm{MIC}$ for Cryptococcus neoformans) when compared with the growth control $(P<0.05)$. Regrowth of fungi was also determined in all the treated groups that showed a fungicidal effect below the detection limit of 10 c.f.u. $\mathrm{ml}^{-1}$. It was also observed that the killing effect of this combination was concentration dependent as well as species dependent. Moreover,

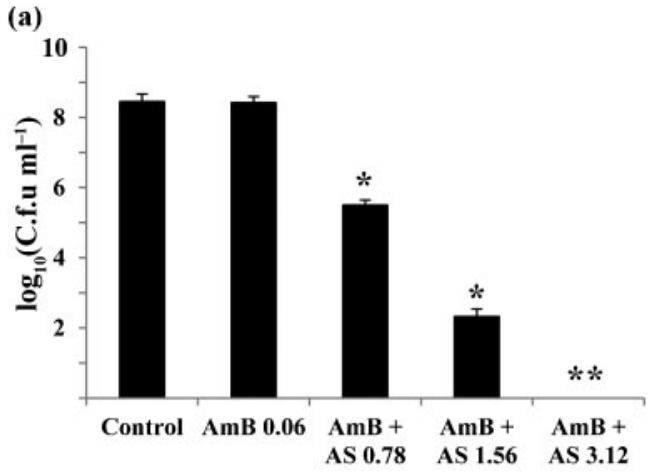

(b)

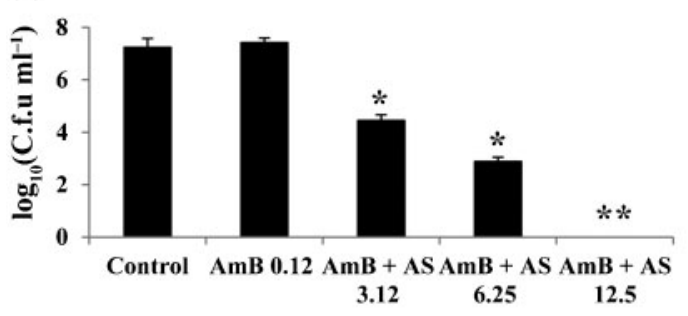

(c)

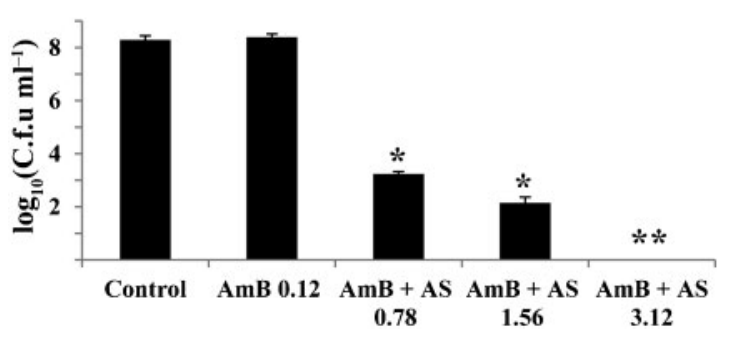

Fig. 2. Effect of the combination of $A m B$ at a fixed concentration of $0.25 \times \mathrm{MIC}(0.125 \times \mathrm{MIC}$ for Cryptococcus neoformans) and in combination with increasing concentrations of acteoside (AS) ranging from 0.78 to $12.5 \mu \mathrm{g} \mathrm{ml}^{-1}$ (as indicated in the figure) on the growth of Candida albicans ATCC 90028 (a), Aspergillus fumigatus MTCC 1811 (b) and Cryptococcus neoformans ATCC 204092 (c). The cultures were incubated in Penassay broth buffered to $\mathrm{pH} 7.0$ for $24 \mathrm{~h}$ at $35{ }^{\circ} \mathrm{C}$. The lower limit of accurate and reproducible quantification was 10 c.f.u. $\mathrm{ml}^{-1}$. Values represent the mean $\log _{10} \pm S D$ of three separate experiments performed in duplicate with similar results each time. ${ }^{*}, P<0.05$; **, $P<0.001$ (Student's $t$-test, compared with the growth control). measurements of untreated conidia of A. fumigatus over time could not be defined accurately because of clumping and the formation of a mycelium, whilst conidia grown in the presence of acteoside did not undergo clumping and were amenable to plating and counting of colonies. The surviving cells from each experiment recovered after $24 \mathrm{~h}$ were again tested for their $\mathrm{AmB}$ and/or acteoside susceptibility. These cells exhibited the same AmB MIC $\left(0.25-1 \mu \mathrm{g} \mathrm{ml}^{-1}\right)$ and exhibited a similar reduction in AmB MIC in the presence of acteoside using the chequerboard technique, with the above-mentioned strains as controls (data not shown).

\section{PAFE studies}

The PAFE of AmB alone and in combination with acteoside was determined against Candida albicans ATCC 90028 and Cryptococcus neoformans ATCC 204092. AmB alone exhibited a PAFE of $1.84 \pm 0.3 \mathrm{~h}$ for Candida albicans at a concentration of $0.25 \times$ MIC $\left.(0.06 \mu \mathrm{g} \mathrm{ml})^{-1}\right)$ and $1.28 \pm 0.6 \mathrm{~h}$ for Cryptococcus neoformans at a concentration $0.125 \times$ MIC $\left(0.12 \mu \mathrm{g} \mathrm{ml} l^{-1}\right)$, respectively. The same concentrations of $\mathrm{AmB}$ in combination with acteoside $\left(3.12 \mu \mathrm{g} \mathrm{m}^{-1}\right)$ resulted in significantly higher PAFEs of $10.01 \pm 0.4 \mathrm{~h}$ for Candida albicans and $18.50 \pm 0.2 \mathrm{~h}$ for Cryptococcus neoformans.

\section{Inhibitory effect of the combination of AmB and acteoside on preformed fungal biofilms}

The combination of $\mathrm{AmB}$ and acteoside exhibited a more prominent reduction in preformed biofilms of Candida albicans ATCC 90028, Cryptococcus neoformans ATCC 204092 and A. fumigatus MTCC 1811 compared with $\mathrm{AmB}$ alone. The results are summarized in Table 2 as 50 and $80 \%$ reductions in the metabolic activities of the biofilms $\left(\mathrm{MBRC}_{50}\right.$ and $\mathrm{MBRC}_{90}$, respectively). The $\mathrm{MBRC}_{50}$ and $\mathrm{MBRC}_{80}$ values of $\mathrm{AmB}$ alone were 4 and $16 \mu \mathrm{g} \mathrm{ml}^{-1}$ for Cryptococcus neoformans, 0.5 and $1 \mu \mathrm{g} \mathrm{ml}^{-1}$ for Candida albicans, and 0.25 and $16 \mu \mathrm{g} \mathrm{m}^{-1}$ for $A$. fumigatus, respectively; these values were reduced by $2-16$ fold in combination with increasing concentrations of acteoside $\left(6.25-25 \mu \mathrm{g} \mathrm{ml}^{-1}\right)$.

\section{Effect of combination of AmB and acteoside on cell viability}

A cell viability assay (determined by c.f.u. count) revealed the extent to which the treated cells of Candida albicans ATCC 90028 were able to survive when removed from exposure to $\mathrm{AmB}$ in the presence or absence of acteoside (Fig. 3a). A $120 \mathrm{~min}$ exposure of cells to a subinhibitory concentration of $\mathrm{AmB}\left(0.25 \mu \mathrm{g} \mathrm{ml}^{-1}\right)$ exhibited a negligible loss in cell viability $(\sim 1 \%)$, whereas the same concentration of $\mathrm{AmB}$ in combination with a fixed concentration of acteoside $\left(25 \mu \mathrm{g} \mathrm{ml}^{-1}\right)$ resulted in a $\geqslant 90 \%$ decrease in viability with respect to untreated cells $(P<0.05)$. AmB alone was found to be effective at a concentration of $4.0 \mu \mathrm{g}$ 
Table 2. In vitro synergistic effect of acteoside (AS) on the activity of AmB on Candida albicans ATCC 90028, Cryptococcus neoformans ATCC 204092 and Aspergillus fumigatus MTCC 1811 preformed biofilms

Biofilms formed in 96-well microtitre plates were quantified using an XTT reduction assay at an absorbance of $490 \mathrm{~nm}$. The results shown are from one of three separate experiments with two replicates; similar results were observed each time. The MIC for biofilms is based on the lowest drug concentration producing 50 and $80 \%$ or more reduction in metabolic activity of the entire cellular population relative to the metabolic activity of the untreated growth control, as measured by the XTT reduction assay.

\begin{tabular}{|c|c|c|c|c|c|}
\hline \multirow[t]{2}{*}{ Tested species } & \multirow{2}{*}{$\begin{array}{l}\text { Tested concn of AS } \\
\qquad\left(\mu \mathrm{g} \mathrm{ml}^{-1}\right)\end{array}$} & \multicolumn{4}{|c|}{ MBRC $\left(\mu \mathrm{g} \mathrm{ml}^{-1}\right)$ of $\mathrm{AmB}$ in combination with AS } \\
\hline & & $\mathrm{MBRC}_{50}$ & $\begin{array}{l}\text { Fold reduction in AmB } \\
\text { MIC }\end{array}$ & $\mathrm{MBRC}_{80}$ & $\begin{array}{c}\text { Fold reduction in } \\
\text { AmB MIC }\end{array}$ \\
\hline \multirow{4}{*}{$\begin{array}{l}\text { Candida albicans } \\
\text { ATCC } 90028\end{array}$} & 0 & 0.5 & 0 & 1.0 & 0 \\
\hline & 6.25 & 0.5 & 0 & 1.0 & 0 \\
\hline & 12.5 & 0.25 & 2 & 1.0 & 0 \\
\hline & 25 & 0.12 & 4 & 0.5 & 2 \\
\hline \multirow{4}{*}{$\begin{array}{l}\text { Cryptococcus } \\
\text { neoformans ATCC } \\
204092\end{array}$} & 0 & 4.0 & 0 & 16.0 & 0 \\
\hline & 6.25 & 0.5 & 8 & 4.0 & 4 \\
\hline & 12.5 & 0.25 & 16 & 4.0 & 4 \\
\hline & 25 & 0.25 & 16 & 2.0 & 8 \\
\hline \multirow{4}{*}{$\begin{array}{l}\text { Aspergillus fumigatus } \\
\text { MTCC } 1811\end{array}$} & 0 & 0.25 & 0 & 16.0 & 0 \\
\hline & 6.25 & 0.12 & 2 & 16.0 & 0 \\
\hline & 12.5 & 0.06 & 4 & 8.0 & 2 \\
\hline & 25 & 0.06 & 4 & 8.0 & 2 \\
\hline
\end{tabular}

$\mathrm{ml}^{-1}$ and produced $\geqslant 99.9 \%$ loss of cell viability when compared with the growth control $(P<0.005)$.

\section{Effect of combination of AmB and acteoside on membrane permeability}

PI uptake assay. PI is a small, cationic, membraneimpermeable, fluorescent nucleic acid stain that binds to DNA by intercalating between the bases with little or no sequence preference. Flow cytometric analysis revealed that exposure of a cell suspension of Candida albicans ATCC 90028 to a subinhibitory concentration of $\mathrm{AmB}(0.25 \mu \mathrm{g}$ $\mathrm{ml}^{-1}$ ) did not increase the cell permeability to PI, whereas the same concentration of $\mathrm{AmB}$ in combination with a fixed concentration of acteoside $\left(25 \mu \mathrm{g} \mathrm{ml}^{-1}\right)$ increased the cell permeability to PI due to disruption of membrane integrity. This resulted in an increase in fluorescence in comparison with the untreated control (Fig. 3b).

Leakage of $260 \mathrm{~nm}$-absorbing material. The loss of membrane integrity of the cell suspension of Candida albicans ATCC 90028 was further confirmed by detection of leakage of $260 \mathrm{~nm}$-absorbing material. The cell suspension exposed to the combination of $\mathrm{AmB}(0.25 \mu \mathrm{g}$ $\left.\mathrm{ml}^{-1}\right)$ and acteoside $\left(25 \mu \mathrm{g} \mathrm{ml} l^{-1}\right)$ resulted in increased release of $260 \mathrm{~nm}$-absorbing material, which was comparable to that of $\mathrm{AmB}$ alone at a concentration of $4.0 \mu \mathrm{g} \mathrm{ml}^{-1}$ when both were compared with the growth control $(P<0.001)$. AmB alone at $0.25 \mu \mathrm{g} \mathrm{ml}^{-1}$ showed insignificant leakage of $260 \mathrm{~nm}$-absorbing material (Fig. 4).

\section{Acute toxicity studies of acteoside}

Toxicity studies were performed to determine the maximum tolerable dose of acteoside after administration of a single-bolus dose ranging from 100 to $2000 \mathrm{mg}$ (kg body weight $)^{-1}$ by the oral or intraperitoneal route to overnightfasted Swiss albino mice $(n=10)$. No adverse pathological symptoms or mortality were observed in the treated animals, and there was no change in general behaviour, when compared with the vehicle-treated group. No death was recorded in the 14 days of the observation period in male or female animals given acteoside at $2000 \mathrm{mg} \mathrm{kg}-1$ either orally or intraperitoneally. The animals did not show any changes in general behaviour or other physiological activities during the observation period.

\section{DISCUSSION}

Acteoside has been studied extensively for its various pharmacological activities. Here, for the first time, we have documented the synergistic activity of $\mathrm{AmB}$ with acteoside, which had no intrinsic antifungal activity $\left(\geqslant 1000 \mu \mathrm{g} \mathrm{ml}^{-1}\right)$. The data demonstrated that acteoside significantly reduced the inhibitory concentration of $\mathrm{AmB}$ against clinically important fungal pathogens (Cryptococcus neoformans, 
(a)

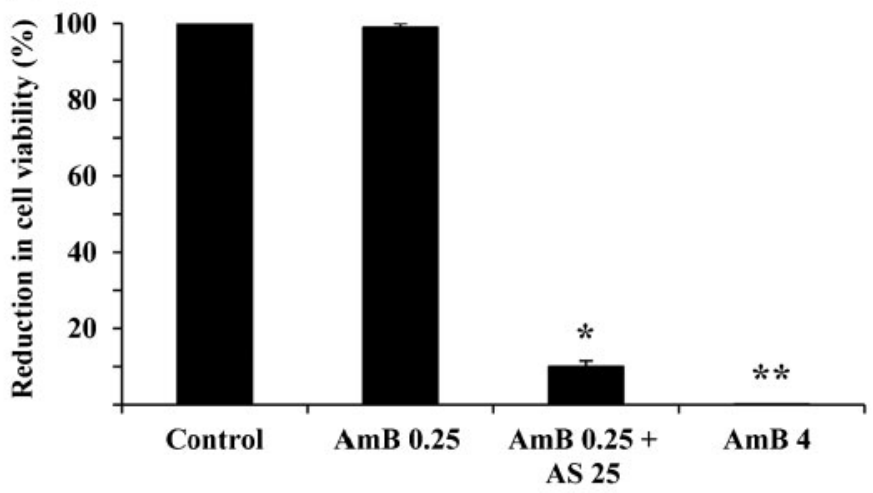

(b)
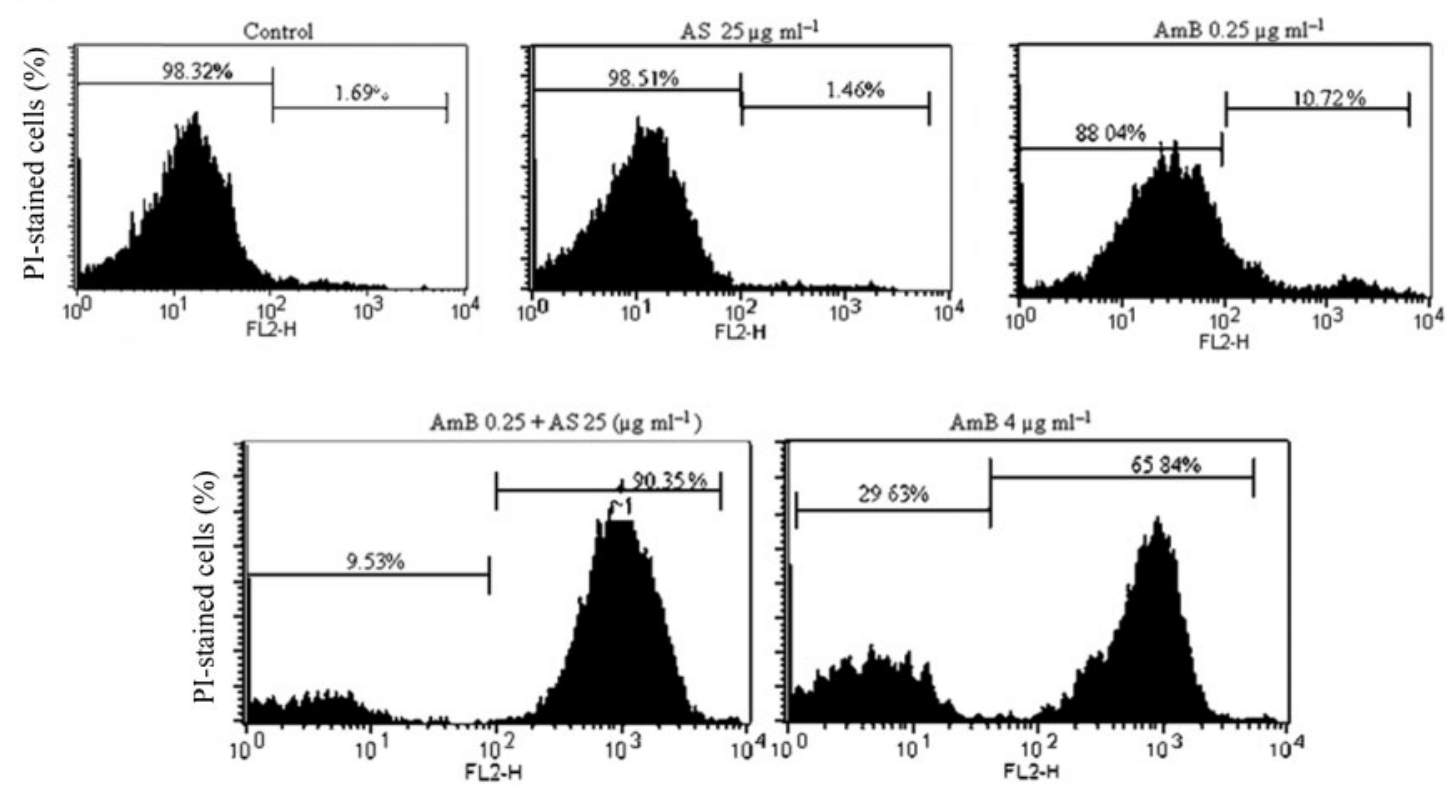

Fig. 3. Effect of $A m B$ in the presence or absence of acteoside (AS) on reduction in cell viability (a) and uptake of $\mathrm{PI}$ (b) in a cell suspension of Candida albicans ATCC 90028 . Cells $\left(\sim 5 \times 10^{7}\right.$ c.f.u. $\left.\mathrm{ml}^{-1}\right)$ were exposed to a subinhibitory concentration of $\mathrm{AmB}\left(0.25 \mu \mathrm{g} \mathrm{ml}^{-1}\right)$ alone and in combination with a fixed concentration of $\mathrm{AS}\left(25 \mu \mathrm{g} \mathrm{ml}^{-1}\right)$ for $120 \mathrm{~min}$ at $35{ }^{\circ} \mathrm{C}$. AmB was also tested alone at a concentration of $4 \mathrm{\mu g} \mathrm{ml}^{-1}$ as the positive control, whilst cells without AmB and AS served as a negative (growth) control. The results of three separate experiments with two replicates are represented (means \pm SD in a) and similar results were observed each time. ${ }^{*}, P<0.05 ;{ }^{* *}, P<0.005$ (Student's $t$-test compared with the growth control).

Candida species and Aspergillus species) as determined by a chequerboard technique. Furthermore, a combination of subinhibitory concentrations of AmB with acteoside exhibited fungicidal activity. This synergistic fungicidal effect was more prominent for Cryptococcus neoformans than for Candida albicans and A. fumigatus. It was also observed that these synergistic fungicidal effects of AmB in combination with acteoside against various other species of fungi were concentration dependent as well as species dependent. In each case, the values of these combinations for the reference strain and clinical isolate showed no significant difference when compared with each other (unpublished data). The combination of $\mathrm{AmB}$ and acteoside could effectively reduce the levels of preformed biofilms of Cryptococcus neoformans, Candida albicans and A. fumigatus. However, AmB alone or in combination with acteoside did not exhibit $>90 \%$ reduction or complete killing (sterility) of the biofilms up to the highest tested concentration of AmB, which was $32 \mu \mathrm{g} \mathrm{ml}^{-1}$ in the present study. This observation regarding the activity of $\mathrm{AmB}$ against these strains was in agreement with the findings of Ramage et al. (2001), Martinez \& Casadevall (2006) and Mowat et al. (2007). Biofilm-associated micro-organisms are refractory to both antimicrobial agents and the host immune response. Even the newer antifungal agents, such as echinocandins and liposomal formulations of $\mathrm{AmB}$, have not been able to 


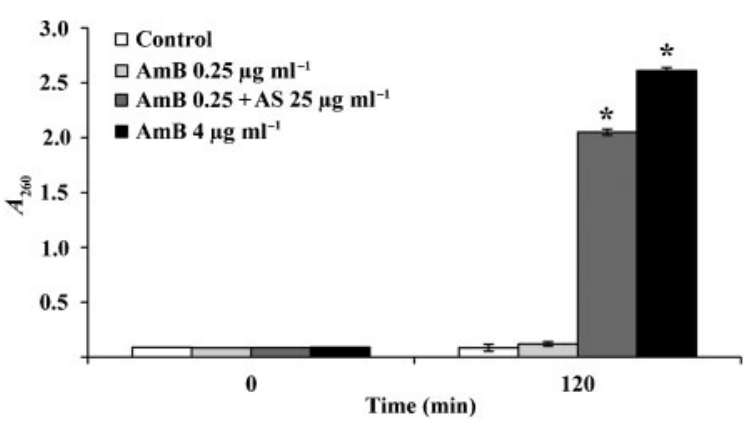

Fig. 4. Effect of $A m B$ in the presence and absence of acteoside (AS) on Candida albicans ATCC 90028, measured by loss of $260 \mathrm{~nm}$-absorbing material. Cells $\left(\sim 5 \times 10^{7}\right.$ c.f.u. $\left.\mathrm{ml}^{-1}\right)$ were exposed to a subinhibitory concentration of AmB $\left(0.25 \mu \mathrm{g} \mathrm{m}^{-1}\right)$ alone and in combination with a fixed concentration of AS $(25 \mu \mathrm{g}$ $\mathrm{ml}^{-1}$ ) for $120 \mathrm{~min}$ at $35{ }^{\circ} \mathrm{C}$. Treatment with AmB at a concentration of $4 \mu \mathrm{g} \mathrm{ml}^{-1}$ followed by sonication was used as a positive control, whilst cells without AmB and AS served as a negative (growth) control. The means \pm SD of two separate experiments with two replicates are represented and similar results were observed each time. $P<0.001$ (Student's $t$-test) was considered to be statistically significant when compared with the growth control.

demonstrate complete eradication of sessile organisms within mature biofilms (Shuford et al., 2007; Pierce et al., 2008; Ferreira et al., 2009).

AmB binds to ergosterol, one of the cell membrane sterols, and damages the cell membrane directly, leading to fungicidal activity. Acteoside potentiated the membranedisruption activity of $\mathrm{AmB}$, as the combination of a subinhibitory concentration of $\mathrm{AmB}$ with acteoside produced loss of cell viability, whereas the same concentrations of AmB and acteoside, when tested individually, did not cause any loss in cell viability. Flow-cytometric analysis of the combination-treated cells revealed enhanced uptake of PI from these cells, indicating that the decrease in viability was accompanied by an increase in cell membrane permeability. Sodium deoxycholate, an anionic detergent, was used in this study, as it is reported to enhance the diffusion of PI across the cell wall to pass through the damaged yeast cell membranes (Ramani et al., 1997; Joung et al., 2007). Sodium deoxycholate alone is non-toxic to Candida albicans, as growth controls do not show dye uptake in the presence of deoxycholate (Ramani et al., 1997). We also found that PI uptake did not correlate completely with cell death. This was more prominent in the case of AmB. A $120 \mathrm{~min}$ exposure of $\mathrm{AmB}$ at a concentration of $4.0 \mu \mathrm{g} \mathrm{ml}^{-1}$ resulted in $\geqslant 99.9 \%$ killing (c.f.u. count below the detection limit of 10 c.f.u. $\mathrm{ml}^{-1}$ ) in Candida albicans, whereas the corresponding PI uptake in this group was $65.84 \%$. Similar observations with AmB have also been reported by Green et al. (1994), who demonstrated that $>90 \%$ of the cells were killed after $3 \mathrm{~h}$ of incubation in $\mathrm{AmB}$ at a concentration of approximately $5 \times$ MIC, but that only $30-40 \%$ of the yeast cells had incorporated PI into their nucleic acids. Cox et al. (2001) also observed similar results with tea tree oil, in which only $54 \%$ of Candida albicans cells were permeable to PI after exposure to the oil in spite of an $84 \%$ reduction in viability. In this study, we were unable to pinpoint the exact mechanism of action of this combination and can only speculate. Acteoside is reported to be a potent antioxidant (Li et al., 2008; Saxena et al., 2010). The subinhibitory concentration of AmB probably enhanced the cellular uptake of acteoside and, once inside the cell, this compound may have inhibited one or more physiological or biochemical process. Similar observations were made by Andrews et al. (1977) and Beggs et al. (1978), who found a synergistic action of $\mathrm{AmB}$ and antioxidants. They also speculated that antioxidants protect $\mathrm{AmB}$ from autooxidation, thereby decreasing the rate of spontaneous inactivation and prolonging its biological activity. We believe that the same observation may also be true for our study.

Acteoside alone did not show any acute toxic effect up to a concentration of $2000 \mathrm{mg} \mathrm{kg}^{-1}$ by the oral or intraperitoneal route. The cytotoxicity profile of this compound has been determined using a polymorphonuclear leukocyte viability test in Wistar rats and no cytotoxic effect was observed when tested at concentrations up to $1000 \mu \mathrm{g} \mathrm{ml}^{-1}$ (Qazi et al., 2006). The present results showed that acteoside is probably safe for acute use in vivo. Acteoside is a glycoside, and these classes of compounds undergo rapid deglycosylation, thereby decreasing their bioavailability. Intravenous administration of acteoside $\left(3 \mathrm{mg} \mathrm{kg}^{-1}\right)$ in rats showed an impressive $C_{\max }$ (maximum concentration of acteoside in the plasma) of $\sim 48.6 \mu \mathrm{g} \mathrm{ml}^{-1}$ but a short halflife of $10.7 \mathrm{~min}$ ( $\mathrm{Wu}$ et al., 2006). We are of the opinion that, using a suitable formulation approach, the problem of the short half-life of this compound should be addressed and the combination should be tested for in vivo efficacy.

In conclusion, our in vitro data indicate that acteoside shows a potent synergistic fungicidal effect when combined with $\mathrm{AmB}$ against clinically significant fungal species. Such combinations may have translational value in the development of more efficacious and safe drugs for fungal infections.

\section{ACKNOWLEDGEMENTS}

The authors thank the Director of the Indian Institute of Integrative Medicine for his sustained interest and encouragement. I.A. is grateful to the Council of Scientific and Industrial Research (CSIR), New Delhi, India, for the award of a senior research fellowship [31/21/ 67 (2008)-EMR-I] and for providing us with the financial support to carry out this study (P-81101). We are also thankful to Dr Fayaz Ahmed Malik of our institute for flow cytometry support.

\section{REFERENCES}

Afeltra, J. \& Verweij, P. E. (2003). Antifungal activity of nonantifungal drugs. Eur J Clin Microbiol Infect Dis 22, 397-407. 
Andrews, F. A., Beggs, W. H. \& Sarosi, G. A. (1977). Influence of antioxidants on the bioactivity of amphotericin B. Antimicrob Agents Chemother 11, 615-618.

Antoniadou, A. \& Giamarellou, H. (2007). Fever of unknown origin in febrile leukopenia. Infect Dis Clin North Am 21, 1055-1090, x.

Beggs, W. H., Andrews, F. A. \& Sarosi, G. A. (1978). Synergistic action of amphotericin B and antioxidants against certain opportunistic yeast pathogens. Antimicrob Agents Chemother 13, 266-270.

Berenbaum, M. C. (1978). A method for testing for synergy with any number of agents. J Infect Dis 137, 122-130.

Chopra, R. N., Nayar, S. L. \& Chopra, I. C. (1956). Glossary of Indian Medicinal Plants. New Delhi: Council of Scientific and Industrial Research.

CLSI (2008a). Reference Method for Broth Dilution Antifungal Susceptibility Testing of Yeasts. Approved standard M27-A3. Wayne, PA: Clinical and Laboratory Standards Institute.

CLSI (2008b). Reference Method for Broth Dilution Antifungal Susceptibility Testing of Filamentous Fungi. Approved standard M38-A2. Wayne, PA: Clinical and Laboratory Standards Institute.

Cox, S. D., Mann, C. M., Markham, J. L., Gustafson, J. E., Warmington, J. R. \& Wyllie, S. G. (2001). Determining the antimicrobial actions of tea tree oil. Molecules 6, 87-91.

Craig, W. A. \& Gudmundsson, S. (1996). Postantibiotic effect. In Antibiotics in Laboratory Medicine, 4th edn, pp. 296-329. Edited by V. Lorian. Baltimore, MD: Williams and Wilkins Co.

CSIR (1956). The Wealth of India: a Dictionary of Indian Raw Materials and Industrial Products, vol. 2. New Delhi: Council of Scientific and Industrial Research.

Díaz, A. M., Abad, M. J., Fernández, L., Silván, A. M., De Santos, J. \& Bermejo, P. (2004). Phenylpropanoid glycosides from Scrophularia scorodonia: in vitro anti-inflammatory activity. Life Sci 74, 2515-2526.

Eliopoulus, G. M. \& Moellering, R. C. J. (1996). Antimicrobial combinations. In Antibiotics in Laboratory Medicine, 4th edn, pp. 330396. Edited by V. Lorian. Baltimore, MD: Williams and Wilkins Co.

Ferreira, J. A. G., Carr, J. H., Starling, C. E. F., de Resende, M. A. \& Donlan, R. M. (2009). Biofilm formation and effect of caspofungin on biofilm structure of Candida species bloodstream isolates. Antimicrob Agents Chemother 53, 4377-4384.

Green, L., Petersen, B., Steimel, L., Haeber, P. \& Current, W. (1994). Rapid determination of antifungal activity by flow cytometry. J Clin Microbiol 32, 1088-1091.

Hemaiswarya, S., Kruthiventi, A. K. \& Doble, M. (2008). Synergism between natural products and antibiotics against infectious diseases. Phytomedicine 15, 639-652.

Joung, Y. H., Kim, H. R., Lee, M. K. \& Park, A. J. (2007). Fluconazole susceptibility testing of Candida species by flow cytometry. J Infect 54, 504-508.

Khan, I. A., Mirza, Z. M., Kumar, A., Verma, V. \& Qazi, G. N. (2006). Piperine, a phytochemical potentiator of ciprofloxacin against Staphylococcus aureus. Antimicrob Agents Chemother 50, 810-812.

Kumar, A., Khan, I. A., Koul, S., Koul, J. L., Taneja, S. C., Ali, I., Ali, F., Sharma, S., Mirza, Z. M. \& other authors (2008). Novel structural analogues of piperine as inhibitors of the NorA efflux pump of Staphylococcus aureus. J Antimicrob Chemother 61, 1270-1276.

Lechner, D., Gibbons, S. \& Bucar, F. (2008). Plant phenolic compounds as ethidium bromide efflux inhibitors in Mycobacterium smegmatis. J Antimicrob Chemother 62, 345-348.

Li, L., Tsao, R., Yang, R., Liu, C., Young, J. C. \& Zhu, H. (2008). Isolation and purification of phenylethanoid glycosides from Cistanche deserticola by high-speed counter-current chromatography. Food Chem 108, 702-710.
Malla, B. \& Chhetri, R. B. (2009). Indigenous knowledge on ethnobotanical plants of Kavrepalanchowk District. Kathmandu University J Sci Eng Tech. 5, 96-109.

Marchetti, O., Moreillon, P., Glauser, M. P., Bille, J. \& Sanglard, D. (2000). Potent synergism of the combination of fluconazole and cyclosporine in Candida albicans. Antimicrob Agents Chemother 44, 2373-2381.

Mariné, M., Espada, R., Torrado, J., Pastor, F. J. \& Guarro, J. (2008). Efficacy of a new formulation of amphotericin B in a murine model of disseminated infection by Candida glabrata. J Antimicrob Chemother 61, 880-883.

Marr, K. A., Boeckh, M., Carter, R. A., Kim, H. W. \& Corey, L. (2004). Combination antifungal therapy for invasive aspergillosis. Clin Infect Dis 39, 797-802.

Martinez, L. R. \& Casadevall, A. (2006). Susceptibility of Cryptococcus neoformans biofilms to antifungal agents in vitro. Antimicrob Agents Chemother 50, 1021-1033.

Mowat, E., Butcher, J., Lang, S., Williams, C. \& Ramage, G. (2007). Development of a simple model for studying the effects of antifungal agents on multicellular communities of Aspergillus fumigatus. J Med Microbiol 56, 1205-1212.

Newman, D. J. \& Cragg, G. M. (2007). Natural products as sources of new drugs over the last 25 years. J Nat Prod 70, 461-477.

Nucci, M. \& Perfect, J. R. (2008). When primary antifungal therapy fails. Clin Infect Dis 46, 1426-1433.

Odds, F. C. (2003). Synergy, antagonism, and what the chequerboard puts between them. J Antimicrob Chemother 52, 1.

OECD (2006). OECD Guidelines for Testing of Chemicals, Guideline 425: Acute Oral Toxicity - Up-And-Down Procedure. Paris: Organization for Economic Cooperation and Development.

Ostrosky-Zeichner, L., Marr, K. A., Rex, J. H. \& Cohen, S. H. (2003). Amphotericin B: time for a new "gold standard". Clin Infect Dis 37, 415-425.

Pfaller, M. A., Sheehan, D. J. \& Rex, J. H. (2004). Determination of fungicidal activities against yeasts and molds: lessons learned from bactericidal testing and the need for standardization. Clin Microbiol Rev 17, 268-280.

Pierce, C. G., Uppuluri, P., Tristan, A. R., Wormley, F. L., Jr, Mowat, E., Ramage, G. \& Lopez-Ribot, J. L. (2008). A simple and reproducible 96-well plate-based method for the formation of fungal biofilms and its application to antifungal susceptibility testing. Nat Protoc 3, 14941500.

Qazi, G. N., Suri, O. P., Bedi, K. L., Suri, K. A., Gupta, B. D., Jaggi, B. S., Kapahi, B. K., Satti, N. K., Amina, M. \& other authors (2006). Hepatoprotective agent of plant origin and a process thereof. United States Patent number: US 6,989,162 B2.

Ramage, G., Vande Walle, K., Wickes, B. L. \& López-Ribot, J. L. (2001). Standardized method for in vitro antifungal susceptibility testing of Candida albicans biofilms. Antimicrob Agents Chemother 45, 2475-2479.

Ramani, R., Ramani, A. \& Wong, S. J. (1997). Rapid flow cytometric susceptibility testing of Candida albicans. J Clin Microbiol 35, 2320-2324.

Richardson, M. D. (2005). Changing patterns and trends in systemic fungal infections. J Antimicrob Chemother 56 (Suppl. 1), i5-il1.

Saxena, A., Saxena, A. K., Singh, J. \& Bhushan, S. (2010). Natural antioxidants synergistically enhance the anticancer potential of AP9cd, a novel lignan composition from Cedrus deodara in human leukemia HL-60 cells. Chem Biol Interact 188, 580-590.

Sharma, S., Kumar, M., Sharma, S., Nargotra, A., Koul, S. \& Khan, I. A. (2010). Piperine as an inhibitor of Rv1258c, a putative multidrug efflux pump of Mycobacterium tuberculosis. J Antimicrob Chemother 65, 1694-1701. 
Shuford, J. A., Piper, K. E., Steckelberg, J. M. \& Patel, R. (2007). In vitro biofilm characterization and activity of antifungal agents alone and in combination against sessile and planktonic clinical Candida albicans isolates. Diagn Microbiol Infect Dis 57, 277-281.

Stavri, M., Piddock, L. J. V. \& Gibbons, S. (2007). Bacterial efflux pump inhibitors from natural sources. J Antimicrob Chemother 59, 1247-1260.

Stergiopoulou, T., Meletiadis, J., Sein, T., Papaioannidou, P., Tsiouris, I., Roilides, E. \& Walsh, T. J. (2009). Comparative pharmacodynamic interaction analysis between ciprofloxacin, moxifloxacin and levofloxacin and antifungal agents against Candida albicans and Aspergillus fumigatus. J Antimicrob Chemother 63, 343-348.

Werle, M. (2008). Natural and synthetic polymers as inhibitors of drug efflux pumps. Pharm Res 25, 500-511.

Wilson, L. S., Reyes, C. M., Stolpman, M., Speckman, J., Allen, K. \& Beney, J. (2002). The direct cost and incidence of systemic fungal infections. Value Health 5, 26-34.
Wu, Y.-T., Lin, L.-C., Sung, J.-S. \& Tsai, T.-H. (2006). Determination of acteoside in Cistanche deserticola and Boschniakia rossica and its pharmacokinetics in freely-moving rats using LC-MS/MS. J Chromatogr B Analyt Technol Biomed Life Sci 844, 89-95.

Wu, Y.-T., Tsai, T.-R., Lin, L.-C. \& Tsai, T.-H. (2007). Liquid chromatographic method with amperometric detection to determine acteoside in rat blood and brain microdialysates and its application to pharmacokinetic study. J Chromatogr B Analyt Technol Biomed Life Sci 853, 281-286.

Yang, F., Li, X.-C., Wang, H.-Q. \& Yang, C.-R. (1996). Flavonoid glycosides from Colebrookea oppositifolia. Phytochemistry 42, 867-869.

Zhang, L., Yan, K., Zhang, Y., Huang, R., Bian, J., Zheng, C., Sun, H., Chen, Z., Sun, N. \& other authors (2007). High-throughput synergy screening identifies microbial metabolites as combination agents for the treatment of fungal infections. Proc Natl Acad Sci U S A 104, $4606-4611$. 